\title{
CIÊNCIA'NATURA
}

\section{Avaliação do Desempenho do Modelo WRF para Prognóstico do vento na Região Central de Alagoas - Craíbas}

\section{Performance Evaluation Model WRF for wind forecast in de central region of Alagoas State - Craíbas}

Silvania Maria Santos da Silva ${ }^{1}$; Roberto Fernando da Fonseca Lyra ${ }^{2}$; Rosiberto Salustiano da Silva Junior ${ }^{3}$, Nareida Simone Delgado da $\mathrm{Cruz}^{4}$ e Sâmara dos Santos Silva ${ }^{5}$

${ }^{1}$ Curso de Pós-Graduação em Meteorologia, Maceió, Brasil

sylvanniasantos@hotmail.com

${ }^{2}$ Instituto de Ciências Atmosféricas, Maceió, Brasil

rffl@ccen.ufal.br

${ }^{3}$ Instituto de Ciências Atmosféricas,Maceió, Brasil rosibertojr@gmail.com

${ }^{4}$ Curso de Pós-Graduação em Meteorologia, Maceió, Brasil nareida.esa@gmail.com

${ }^{5}$ Curso de Graduação em Meteorologia, Maceió, Brasil samarass_@hotmail.com

\section{Resumo}

O objetivo deste trabalho foi avaliar o desempenho do modelo WRF para prognóstico da velocidade do vento na região central do Estado de Alagoas (Craíbas). As saídas do modelo (médias horárias) foram comparadas aos dados anemométricos do projeto PVPN (Previsão do Vento em Parques Eólicos no Nordeste Brasileiro). O periodo estudado foi de janeiro a dezembro de 2014. Os resultados mostraram que o modelo WRF estimou bem as médias mensais e diárias da velocidade do vento. Os desvios são maiores nos meses secos (0,66 m.s. $\left.\mathrm{s}^{-1}\right)$ do que nos meses chuvosos. Ao longo de todo o ano o a diferença média (WRFOBS) foi de apenas 0,23 m.s. $\left(6,14 \mathrm{~m} \cdot \mathrm{s}^{-1}\right.$ versus $\left.5,91 \mathrm{~m} \cdot \mathrm{s}^{-1}\right)$, provando que este modelo é uma ótima ferramenta para previsão do vento para fins de aproveitamento eólico.

Palavras-chave: Vento; Energia Eólica; Modelo Atmosférico; Modelo WRF.

\section{Abstract}

The aim of this work was to evaluate the performance of the WRF model for estimate wind speed in the central region of Alagoas State (Brazil). The wind velocity (WRF outputs) were compared with anemometric data of the PVPN projetc (Previsão do Vento em Parques Eólicos no Nordeste Brasileiro "Wind forecast for wind farms in the Brazilian Northeast"), from January to December 2014. The results showed that the WRF model estimated very well the monthly and daily wind speed averages. Deviations are greater in the dry season. Throughout the year the mean difference (WRF-OBS) was only $0.23 \mathrm{~m} . \mathrm{s}^{-1}\left(6.14 \mathrm{~m} . \mathrm{s}^{-1}\right.$ versus $5.91 \mathrm{~m} . \mathrm{s}^{-}$ $\left.{ }^{1}\right)$, showing that this model is a good tool for forecasting wind for wind farms. 


\section{Introdução}

Na matriz energética Brasileira, a geração de energia elétrica é feita basicamente a partir de centrais hidroelétricas chegando a quase três quartos do total. Além de estarmos chegando ao limite do aproveitamento deste tipo de recurso energético, deve ser levada em conta a sua vulnerabilidade às oscilações climáticas. Por outro lado, o Brasil está apenas começando a explorar o seu potencial eólico com produção da ordem de $300 \mathrm{MW}$. O desenvolvimento da energia eólica no Brasil vai ajudar o país a cumprir os objetivos estratégicos de reforçar a segurança energética nacional, gerar mais empregos e, ao mesmo tempo, reduzir as emissões nacionais de gases com efeito de estufa relacionadas com a geração de energia elétrica. O Brasil possui um potencial eólico estimado de 143GW (AMARANTE et al., 2001), no entanto este valor pode ser ainda maior devido ao aumento das dimensões dos aerogeradores e à possibilidade de exploração do potencial offshore.

A região Nordeste do Brasil possui ainda uma particularidade que é a complementaridade hidroeólica, uma vez que as vazões dos rios e o regime de ventos possuem um ciclo sazonal alternado, que somado ao despacho complementar das usinas termoelétricas permite o ajuste à demanda de eletricidade, garantindo a segurança da geração 13 elétrica na região. Este gerenciamento só é possível devido ao Sistema Interligado Nacional (SIN) que permite remanejo das cargas pelo Operador Nacional do Sistema (ONS). Embora com certa defasagem em relação à expansão mundial, nos últimos anos o país vem experimentando um forte crescimento no setor eólico, impulsionado principalmente pelo PROINFA.

De acordo com o Atlas Eólico do Estado de Alagoas (ELETROBRÁS, 2008), o estado apresenta potencial eólico propício (336 MW a 75m de altura, para áreas com ventos equivalentes ou superiores a 7,0 m. $\mathrm{s}^{-1}$ ) e poderá ser aproveitado nos limites de inclusão do sistema elétrico regional.

Neste contexto, se faz cada vez mais necessário o desenvolvimento de uma metodologia para a previsão de energia eólica de curto prazo adequada ao território brasileiro, que seja embasada nas previsões numéricas disponibilizadas operacionalmente no país e compatível com o clima tropical. Nos últimos anos muitos estudos foram realizados utilizando técnicas diversas visando à otimização destas previsões de vento, desde modelagem física em microescala (LANGE, 2006) até ferramentas estatísticas diversas (FAN et al., 2009). Comumente opta-se por uma combinação entre estas técnicas para um melhor desempenho das previsões (ERNST et al., 2007).

Dentre as técnicas de modelagem mais utilizadas em estudos diversos está o modelo WRF (Weather Research and Forecasting), possuindo várias aplicações meteorológicas que permite representar condições atmosféricas reais ou condições projetadas. Por ter grande desempenho na previsão dos dados em termos de parâmetros numéricos, físicos e dinâmicos, o que permite grande adequação ao estudo específico, além de ser um software moderno (SILVA, 2013). Em Alagoas, a avaliação da variabilidade do vento com o uso do modelo WRF tem obtido resultados satisfatórios como mostram alguns autores em seus trabalhos (RAMOS, 2012; MOURA, 2014). Porém de acordo com de FERONI et al. (2013), não possui bom desempenho em ventos de grande intensidade em eventos mais extremos. Neste âmbito, o presente trabalho tem como objetivo avaliar o desempenho do modelo WRF em termos de prognóstico do vento na região central do estado de Alagoas. 


\section{Materiais e Métodos}

A região do agreste alagoano possui um potencial eólico mais propício dentre as outras regiões do Estado. Devido a isto, foi escolhida a área mais central desta região para instalação da torre anemométrica do projeto Previsão do Vento em Parques Eólicos do Nordeste Brasileiro. O sitio experimental (Torre Anemométrica) do projeto fica situada no município de Craíbas $\left(9^{\circ} 37^{\prime}\right.$ de latitude sul e de $36^{\circ} 46^{\prime}$ de longitude oeste).

Foram utilizados os dados anemométricos medidos no sitio experimental do projeto em Craíbas, no ano de 2014 e saídas do WRF (médias horárias). Com relação aos dados observados as medidas foram feitas a cada 15 segundos e armazenados em médias a cada 10 minutos em 3 níveis: 30, 50 e $100 \mathrm{~m}$. No presente trabalho foram utilizados somente os dados do nível $2(50 \mathrm{~m})$ para o qual foram calculadas as médias horárias e diárias. No caso das saídas do WRF foram utilizadas as médias horárias para o nível de $50 \mathrm{~m}$, sendo calculadas também as médias diárias. O período de estudo (janeiro/2014 a dezembro/2014) foi escolhido com base na disponibilidade de dados observados da torre anemométrica de Craíbas que fica localizada na região agreste do estado de Alagoas, pois a série de dados para o ano de 2014 se encontrava completa e atual.

No caso das saídas do WRF foram utilizadas as médias horárias para o nível de $50 \mathrm{~m}$, foram calculadas as médias horárias. As simulações do modelo WRF são realizadas em 4 dimensões (x,y,z,t), dado que a malha horizontal repete-se em todos os níveis verticais a cada passo de tempo. A estrutura vertical do modelo é fornecida através da coordenada sigma, que acompanha o contorno do terreno. Para esse estudo foram configurados 3 domínios: o domínio 1 (domínio-mãe) apresenta resolução espacial de $80 \mathrm{~km} \mathrm{e}$ abrange parte da América do Sul e Oceano Atlântico; o domínio 2 tem resolução de 20 km e abrange o NEB e parte do Oceano Atlântico; o domínio 3 possui resolução de 5 km e compreende o Estado de Alagoas.

\section{Resultados}

\subsection{Comparação do WRF com os dados observados.}

Será apresentada a comparação entre a velocidade do vento observada com aquelas simuladas pelo WRF, através das médias mensais e das médias diárias para o ano de estudo (2014).

A Tabela 1 apresenta a estatística das velocidades para cada mês com média, desvio padrão, coeficiente de variação e o coeficiente de correlação linear.

Ao longo de todo o ano o WRF superestimou a velocidade do vento em apenas $0,23 \mathrm{~m} \cdot \mathrm{s}^{-1}\left(6,14 \mathrm{~m} \cdot \mathrm{s}^{-1}\right.$ versus $\left.5,91 \mathrm{~m} \cdot \mathrm{s}^{-1}\right)$. As médias mensais são muito próximas, a diferença varia de $0,02 \mathrm{~m} \cdot \mathrm{s}^{-1} \mathrm{em}$ janeiro a 0,71 $\mathrm{m} . \mathrm{s}^{-1} \mathrm{em}$ novembro. Somente em três meses os desvios foram negativos (fevereiro, março e abril).

A variabilidade foi avaliada através dos coeficientes de variação $(\mathrm{CV})$. O fato dos CVs serem muito próximos foi de alguma forma surpreendente, pois esperava-se que fossem maiores para os dados do WRF. As médias anuais foram idênticas (13\%). Março teve a menor variabilidade (8\%) e junho a maior (19\%), ambos na série de dados WRF.

Em termo de concordância (correlação) não existe uma tendência ou padrão e os coeficientes não são muito altos. O mês de janeiro apresentou a pior correlação linear inclusive negativa $(-0,15)$ e o mês de novembro a melhor $(0,87)$. 
Tabela 1 - Estatística das velocidades para o ano de 2014.

\begin{tabular}{cccccccc}
\hline MÊS & \multicolumn{2}{c}{ MÉD } & \multicolumn{2}{c}{ DP } & \multicolumn{2}{c}{ CV } & CL \\
& WRF & OBS & WRF & OBS & WRF & OBS & \\
\hline Janeiro & $\mathbf{6 , 7 4}$ & $\mathbf{6 , 7 2}$ & $\mathbf{0 , 6 9}$ & $\mathbf{1 , 2 2}$ & $\mathbf{1 0 \%}$ & $\mathbf{1 8 \%}$ & $\mathbf{- 0 , 1 5}$ \\
Fevereiro & $\mathbf{6 , 4 2}$ & $\mathbf{6 , 5 1}$ & $\mathbf{0 , 5 6}$ & $\mathbf{0 , 5 8}$ & $\mathbf{9 \%}$ & $\mathbf{9 \%}$ & $\mathbf{0 , 3 3}$ \\
Março & $\mathbf{6 , 2}$ & $\mathbf{6 , 2 4}$ & $\mathbf{0 , 4 8}$ & $\mathbf{0 , 5 6}$ & $\mathbf{8 \%}$ & $\mathbf{9 \%}$ & $\mathbf{0 , 6 9}$ \\
Abril & $\mathbf{5 , 0 3}$ & $\mathbf{5 , 2 7}$ & $\mathbf{0 , 8 1}$ & $\mathbf{0 , 7 5}$ & $\mathbf{1 6 \%}$ & $\mathbf{1 4 \%}$ & $\mathbf{0 , 5 9}$ \\
Maio & $\mathbf{5 , 1 3}$ & $\mathbf{4 , 6}$ & $\mathbf{0 , 7 1}$ & $\mathbf{0 , 5 2}$ & $\mathbf{1 4 \%}$ & $\mathbf{1 1 \%}$ & $\mathbf{0 , 5}$ \\
Junho & $\mathbf{5 , 2 4}$ & $\mathbf{5 , 1 5}$ & $\mathbf{1 , 0 1}$ & $\mathbf{0 , 9 1}$ & $\mathbf{1 9 \%}$ & $\mathbf{1 8 \%}$ & $\mathbf{0 , 7 7}$ \\
Julho & $\mathbf{5 , 6}$ & $\mathbf{5 , 2 7}$ & $\mathbf{0 , 8 6}$ & $\mathbf{0 , 9 7}$ & $\mathbf{1 5 \%}$ & $\mathbf{1 8 \%}$ & $\mathbf{0 , 8 2}$ \\
Agosto & $\mathbf{5 , 7}$ & $\mathbf{5 , 6 6}$ & $\mathbf{0 , 7 9}$ & $\mathbf{0 , 8 7}$ & $\mathbf{1 4 \%}$ & $\mathbf{1 5 \%}$ & $\mathbf{0 , 6 8}$ \\
Setembro & $\mathbf{6 , 4 1}$ & $\mathbf{6 , 2 2}$ & $\mathbf{0 , 8 1}$ & $\mathbf{0 , 6 1}$ & $\mathbf{1 3 \%}$ & $\mathbf{1 0 \%}$ & $\mathbf{0 , 6 7 2}$ \\
Outubro & $\mathbf{7 , 3}$ & $\mathbf{6 , 6 9}$ & $\mathbf{0 , 7 6}$ & $\mathbf{0 , 5 1}$ & $\mathbf{1 0 \%}$ & $\mathbf{8 \%}$ & $\mathbf{0 , 5 2}$ \\
Novembro & $\mathbf{6 , 8}$ & $\mathbf{6 , 0 9}$ & $\mathbf{1 , 0 5}$ & $\mathbf{1 , 0 4}$ & $\mathbf{1 5 \%}$ & $\mathbf{1 7 \%}$ & $\mathbf{0 , 8 7}$ \\
Dezembro & $\mathbf{7 , 0 8}$ & $\mathbf{6 , 5 2}$ & $\mathbf{0 , 7 2}$ & $\mathbf{0 , 7 1}$ & $\mathbf{1 0 \%}$ & $\mathbf{1 1 \%}$ & $\mathbf{0 , 7 8}$ \\
\hline
\end{tabular}

Para cada mês, foram calculados os desvios médios e máximos (figura 1). Não se verifica nenhum padrão ou ciclo sazonal. Na média, os desvios são maiores nos meses secos $\left(0,66 \mathrm{~m} \cdot \mathrm{s}^{-1}\right)$ do que nos meses chuvosos $\left(0,57 \mathrm{~m} \cdot \mathrm{s}^{-1}\right)$. No estudo de Ramos et al. (2013), desvios mensais oscilaram entre 3 a $5 \mathrm{~m} \cdot \mathrm{s}^{-1}$, nos meses chuvosos nos municípios de Feliz Deserto e Roteiro, no qual o modelo demonstrou um valor superior, por conta da dificuldade que o modelo apresentou em previsões nesse período do ano. Mostrando que o modelo precisava ser mais testado e avaliado para melhorar a previsão em períodos chuvosos.

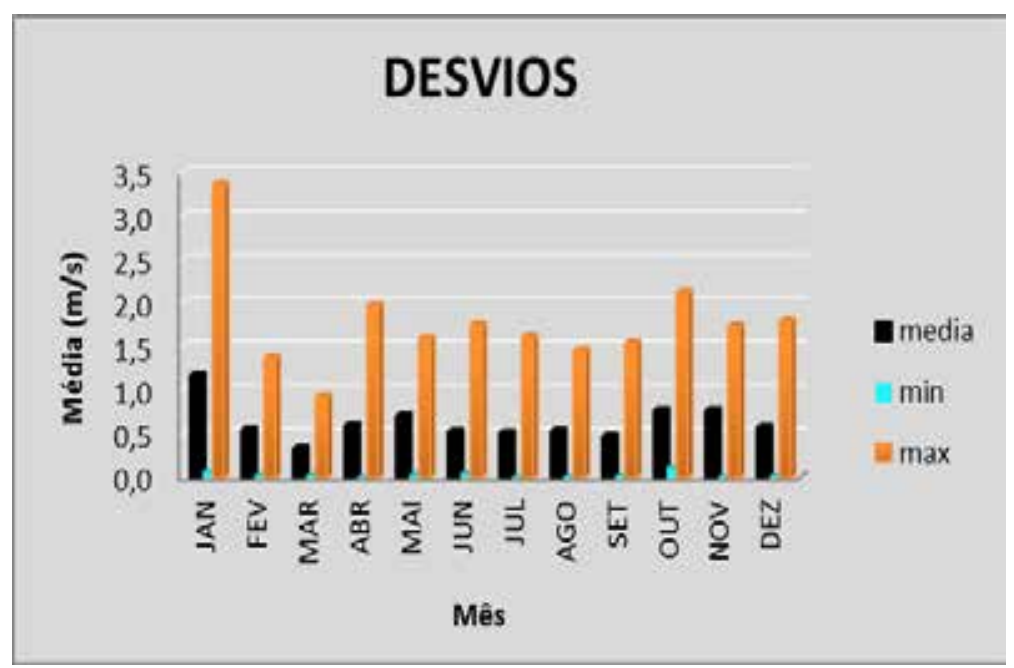

Figura 1 - Médias dos desvios mensais (em preto), máximos mensais (em laranja) e mínimos (em azul) para o ano de 2014.

Confrontando os casos de subestimativa com os de superestimativa (tabela 2) verifica-se que, no geral, o modelo superestimou em 234 dias, e subestimou em 132 dias no ano de 2014. Em apenas três meses, o número de casos de subestimativa mostrou-se superior (fevereiro, março e abril). O mês com o maior número de casos de superestimativa foi dezembro (29) e o com menor abril (8). No caso das subestimativas o mês com maior número foi abril (22) e com menor dezembro (2). 
Tabela 2 - Total de casos de Superestimativa e de subestimativa para cada mês. Craíbas, 2014.

\begin{tabular}{|c|c|c|c|c|c|c|c|c|c|c|c|c|}
\hline $\begin{array}{l}\text { ESTIMATIVA DO } \\
\text { MODELO }\end{array}$ & JAN & FEV & MAR & ABR & MAI & JUN & JUL & AGO & SET & OUT & NOV & DEZ \\
\hline SUPERESTIMATIVA & 18 & 12 & 10 & 8 & 24 & 19 & 25 & 19 & 18 & 26 & 26 & 29 \\
\hline SUBESTIMATIVA & 13 & 17 & 21 & 22 & 7 & 11 & 6 & 12 & 12 & 5 & 4 & 2 \\
\hline
\end{tabular}

\section{Conclusão}

O desempenho do modelo WRF para prever a velocidade do vento na região central do Estado de Alagoas, foi avaliado com êxito. Constatou-se que e constatou-se que o WRF estimou com acurácia as médias mensais e diárias da velocidade do vento e que a margem de erro aumenta para estimativa de médias de períodos mais curtos como médias horárias. Na média, os desvios são maiores nos meses secos $\left(0,66 \mathrm{~m} \cdot \mathrm{s}^{-}\right.$ $\left.{ }^{1}\right)$ do que nos meses chuvosos $\left(0,57 \mathrm{~m} \cdot \mathrm{s}^{-1}\right)$, mostrando que o modelo precisava ser mais testado e avaliado para melhorar a previsão em períodos chuvosos. Em termo de correlação, o mês de janeiro apresentou a pior correlação linear $(-0,15)$. Ao longo de todo o ano o WRF superestimou a velocidade do vento em apenas 0,23 $\mathrm{m} \cdot \mathrm{s}^{-1}\left(6,14 \mathrm{~m} \cdot \mathrm{s}^{-1}\right.$ versus $\left.5,91 \mathrm{~m} \cdot \mathrm{s}^{-1}\right)$. Provando ser um ótimo método para previsão do vento e uma grande ferramenta para o aproveitamento eólico.

\section{Referências}

AMARAnTe, O. A. C.; DA Silva, F. J. L. Atlas do Potencial Eólico Brasileiro. Camargo Schubert Engenharia Eólica, Centro de Pesquisas de Energia Elétrica/CEPEL. Brasília, 2001.

ELETROBRÁS. Atlas Eólico do Estado de Alagoas. 2008. Disponível em: <http:// Atlas\%20Eólico\%20de\%20Alagoas_internet.pdf >. Acesso em: Acessado em 13 Set. 2017.

ERNST, B.; OAKLEAF, B.; AHLSTRON, M. L.; LANGE, M.; MOEHRLEN, C.; LANGE, B. et al.. Predicting the Wind. Vol. 5. n. 6, pp. 78-89. IEEE Power \& Energy magazine, 2007.

FAN, S. L.; YOKOYAMA, J. R.; CHEN, R. L.; LEE, W. J. Forecasting the wind generation using a twostage network based on meteorological information. vol. 24, n. 2, 2009. IEEE Transactions on Energy Conversion, 2009.

FERONI, R. C.; GALVÃO, E. S.; SANTIAGO, A. M.; ALBUQUERQUE, T. T. Análise do desempenho do Modelo WRF na Previsão de Evento Severo em Vitória-ES.” Ciência \& Natura, p. 473-475, 2013.

LANGE, M.; FOCKEN, U. Physical approach to short-term wind power prediction. (pp. 1-208) Berlin: Springer, 2006.

MOURA, J. D. O.; LYRA, R. F. F.; RAMOS, D. N. .S.; IHADUA, I. M. T. J. Simulação do vento utilizando o Modelo WRF com diferentes esquemas de parametrização. 2011, Alagoas. Ed. Suplementar. 
RAMOS, D. R. S. Mapeamento eólico do Estado de Alagoas utilizando ferramentas computacionais e dados observados. 2012, 130 f. Dissertação (Mestrado em Meteorologia) - Universidade Federal de Alagoas (UFAL), Maceió, 2012.

SILVA, A. F. G. S. Avaliação do modelo WRF ao perfil do vento no Centro de Lançamento de Alcântara. 2013. 100 f. Dissertação (Mestrado em Meteorologia) - Instituto Nacional de Pesquisas Espaciais, São José dos Campos, 2013.

Silvania Maria Santos da Silvia

Roberto Fernando da Fonseca Lyra

Rosiberto Salustiano da Silva Junior

Nareida Simone Delgado da Cruz

Sâmara dos Santos Silva
Curso de Pós-graduação em Meteorologia, Maceió, Brasil Email: sylvanniasantos@hotmail.com

Instituto de Ciências Atmosféricas, Maceió, Brasil E-mail: rff@@ccen.ufal.br

Instituto de Ciências Atmosféricas,Maceió, Brasil E-mail: rosibertojr@gmail.com

Curso de Pós-Graduação em Meteorologia, Maceió, Brasil E-mail: nareida.esa@gmail.com

Curso de Graduação em Meteorologia, Maceió, Brasil E-mail: samarass@@hotmail.com 